\title{
Note: An ion source for alkali metal implantation beneath graphene and hexagonal boron nitride monolayers on transition metals
}

\author{
de Lima, L H ; Cun, H Y ; Hemmi, A ; Kälin, T ; Greber, T
}

\begin{abstract}
The construction of an alkali-metal ion source is presented. It allows the acceleration of rubidium ions to an energy that enables the penetration through monolayers of graphene and hexagonal boron nitride. $\mathrm{Rb}$ atoms are sublimated from an alkali-metal dispenser. The ionization is obtained by surface ionization and desorption from a hot high work function surface. The ion current is easily controlled by the temperature of ionizer. Scanning Tunneling Microscopy measurements confirm ion implantation.
\end{abstract}

DOI: https://doi.org/10.1063/1.4848936

Posted at the Zurich Open Repository and Archive, University of Zurich

ZORA URL: https://doi.org/10.5167/uzh-92061

Journal Article

Published Version

Originally published at:

de Lima, L H; Cun, H Y; Hemmi, A; Kälin, T; Greber, T (2013). Note: An ion source for alkali metal implantation beneath graphene and hexagonal boron nitride monolayers on transition metals. Review of Scientific Instruments, 84(12):126104.

DOI: https://doi.org/10.1063/1.4848936 


\title{
Note: An ion source for alkali metal implantation beneath graphene and hexagonal boron nitride monolayers on transition metals
}

\author{
L. H. de Lima, ${ }^{1, a)}$ H. Y. Cun, ${ }^{2}$ A. Hemmi, ${ }^{2}$ T. Kälin, ${ }^{2}$ and T. Greber ${ }^{2}$ \\ ${ }^{1}$ Instituto de Física "Gleb Wataghin", Universidade Estadual de Campinas, 13083-859, Campinas, SP, Brazil \\ ${ }^{2}$ Physik-Institut, Universität Zürich, Winterthurerstrasse 190, CH-8057 Zürich, Switzerland
}

(Received 23 September 2013; accepted 2 December 2013; published online 16 December 2013)

\begin{abstract}
The construction of an alkali-metal ion source is presented. It allows the acceleration of rubidium ions to an energy that enables the penetration through monolayers of graphene and hexagonal boron nitride. $\mathrm{Rb}$ atoms are sublimated from an alkali-metal dispenser. The ionization is obtained by surface ionization and desorption from a hot high work function surface. The ion current is easily controlled by the temperature of ionizer. Scanning Tunneling Microscopy measurements confirm ion implantation. (C) 2013 AIP Publishing LLC. [http://dx.doi.org/10.1063/1.4848936]
\end{abstract}

Recently, monolayers of hexagonal boron nitride (h-BN) and graphene are getting intensive attention due to their capacity for self-assembling of atoms and molecules. ${ }^{1,2}$ The growth of these monolayers on substrates with lattice parameters different from the $\mathrm{sp}^{2}$ monolayer produces superstructures with an in-plane lattice constant in the order of a few nanometers. This is the case, for example, for graphene grown on $\mathrm{SiC}(0001)^{3}$ or hexagonal boron nitride on $\mathrm{Rh}(111){ }^{4}$ These superstructures may be modified by molecular self assembly, ${ }^{2,5}$ or intercalation of hydrogen, for example. ${ }^{6,7} \mathrm{Re}-$ cently, it was demonstrated that noble gas ions of appropriate energy may be immobilized at room temperature between the $\mathrm{sp}^{2}$ layer $^{8}$ and the substrate or cut graphene. ${ }^{9}$ For this purpose, the atoms must be accelerated against the surface to an energy which allows to break the strong $\sigma$ bonds (in the order of $10 \mathrm{eV}$ )

The immobilization of single atoms beneath an ultimately thin $\mathrm{sp}^{2}$ hybridized layer, in so called "nanotents," is an interesting way to functionalize a surface and should, in principle, not be limited to noble gas atoms. Here we show the formation of nanotents by exposing a h$\mathrm{BN} / \mathrm{Rh}(111)$ nanomesh to $\mathrm{Ab}^{+}$ion beam with appropriate energy.

For atoms, the necessary kinetic energy to penetrate a monolayer of graphene or h-BN can be estimated from the displacement threshold energies $T_{d}^{\alpha}$ where $\alpha$ is index for $\mathrm{B}, \mathrm{N}$, or $\mathrm{C}$, atoms, respectively. This threshold energy is the minimum kinetic energy that an atom needs to absorb in order to be ejected from the system. If this energy is provided in a head on elastic collision ${ }^{10}$ with another atom, the kinetic energy of the incident atom like $\mathrm{Rb}$ is given by $E_{k i n}^{R b}=(1+\beta)^{2} T_{d}^{\alpha} / 4 \beta$, where $\beta=\left(m_{\alpha} / m_{R b}\right)$ is the atomic mass ratio, with $\alpha=\mathrm{B}, \mathrm{N}$, or $\mathrm{C}$.

With the values of $T_{d}^{\alpha}$ from first principles, ${ }^{11} E_{k i n}^{R b}$ is approximately $50 \mathrm{eV}$ for both h-BN and graphene.

In order to accelerate the $\mathrm{Rb}$ atoms, it is first necessary to ionize them. For this purpose, we apply the concept of surface

a)lhlima@ifi.unicamp.br ionization. ${ }^{12}$ Basically, it consists in ionizing an atom and desorbing it from a surface with a higher work function $\Phi$ than the ionization potential $I_{P}$ of the atom. ${ }^{13}$ Furthermore, it is necessary that the surface is hot enough such that the ions can desorb thermally. When an atom or molecule approaches a metallic surface, its valence level broadens and lowers its energy towards the Fermi level of the metal. When they overlap the valence electron of the atom may transfer to the Fermi sea of the substrate and an ionized atom is left. The ratio number of ions and neutral atoms on the surface is described by the Saha-Langmuir equation, which predicts the degree of ionization to be proportional to $\exp \left[\left(\Phi-I_{P}\right) / k_{B} T\right]$, where $k_{B}$ is the Boltzmann constant and $T$ is the equilibrium temperature of the system. We first tried Mo $(\Phi=4.6 \mathrm{eV})$ in order to ionize $\mathrm{Rb}$ atoms $\left(I_{P}=4.2 \mathrm{eV}\right)$. This works, although some improvements were achieved with a sputter coated $10 \mathrm{~nm}$ Pt layer on the Mo surface due to the increase of the work function. Basically, the ion current with Pt layer is ten times larger, but the general behavior is the same as reported here for the bare Mo surface.

The inverse process, i.e., charge transfer from the metal to the atom or molecule can occur if the surface has a lower work function than the affinity level of the atom.

Figure 1 shows the scheme of the "head" part of the ion source that is mounted on a CF40 flange. The atoms are evaporated from an alkali-metal (SAES) getter source. ${ }^{14}$ The activation of the $\mathrm{Rb}$ getter is obtained by an electric heating current $\left(I_{R b}\right)$. The advantage of this source relies on the fact that it generates a $\mathrm{Rb}$ vapor that is pure, combining with a precise evaporation rate that is controlled by the current $I_{R b}$ (see Figure 2). The Mo is heated by a Ta filament with the current $I_{F I L}$. After surface ionization, the $\mathrm{Rb}^{+}$ions are accelerated to the potential $U_{M o}$, which determines the beam energy.

Figure 2(a) shows the ion current as a function of $I_{R b}$. The ion current is detected by a screen located approximately $100 \mathrm{~mm}$ from the exit of the ion source. The surface temperature was kept at $409^{\circ} \mathrm{C}$ and the beam energy was $100 \mathrm{eV}$.

Figure 2(a) shows that the evaporation starts at approximately $I_{R b}=5.0 \mathrm{~A}$, which agrees well with the values given by the manufacturer $(5.3 \pm 0.2 \mathrm{~A})^{14}$. Figure $2(\mathrm{~b})$ shows 


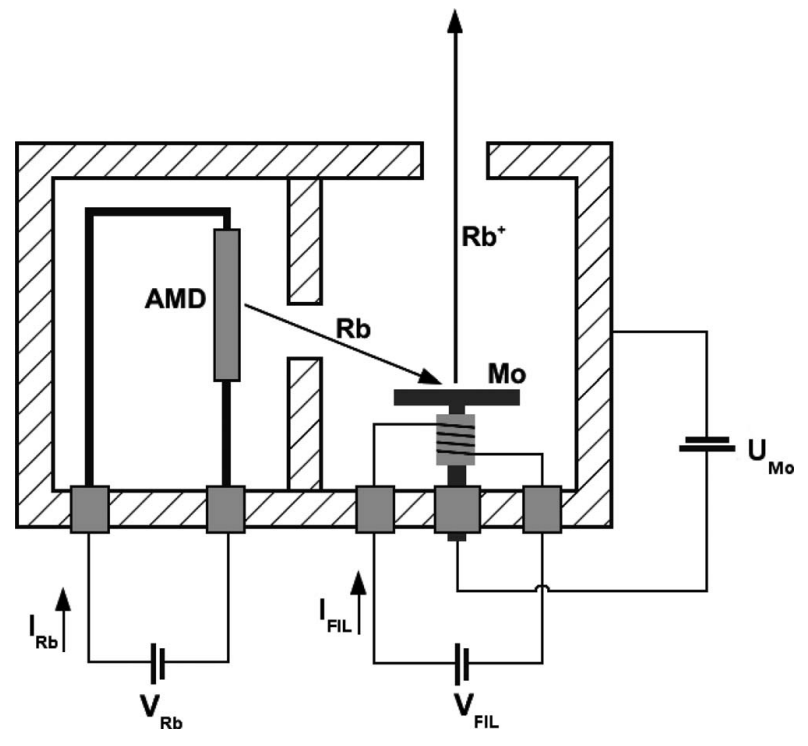

FIG. 1. Scheme of the "head" part of the ion source. The atoms are evaporated from an Alkali Metal Dispenser (AMD), ${ }^{14}$ and ionized on a hot Mo (or $\mathrm{Pt}$ ) surface and then accelerated to the potential $U_{M o}$.
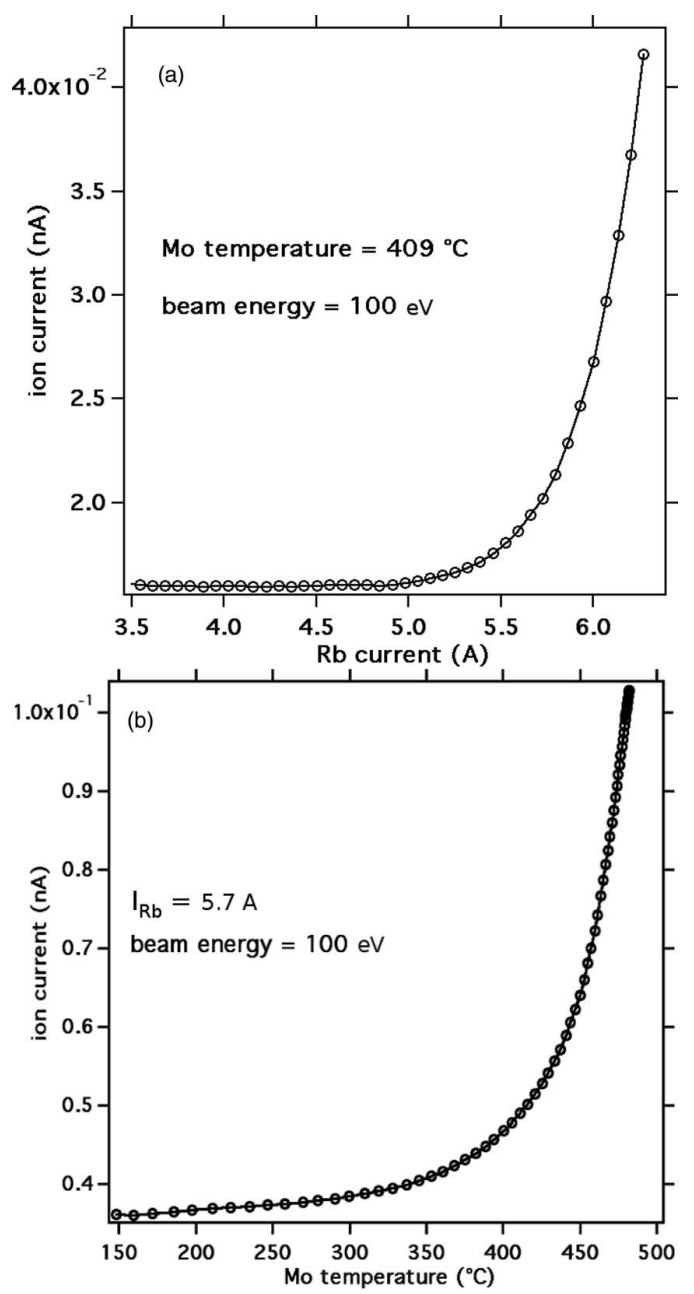

FIG. 2. Performance of the Rb ion source. (a) The evaporation starts at approximately $I_{R b}=5.0 \mathrm{~A}$. (b) Dependence of ion current as a function of the temperature of the Mo.
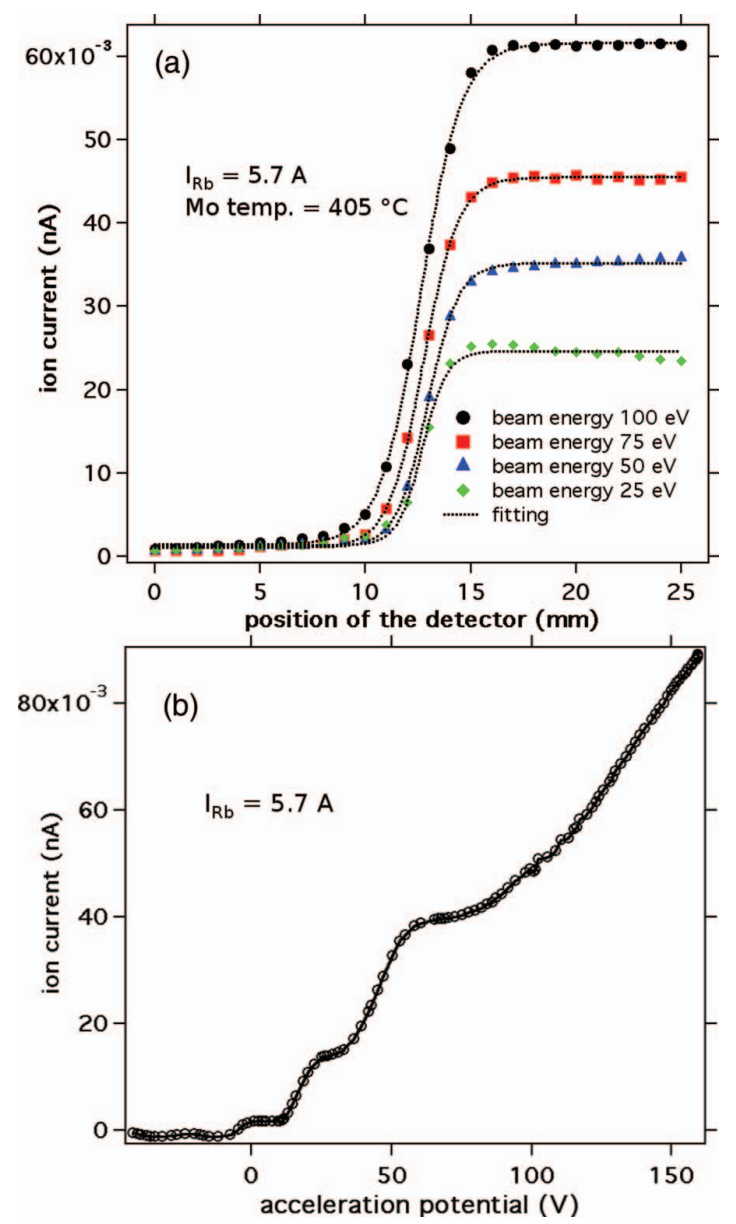

FIG. 3. (a) Ion current as a function of the relative position of the detector (screen). There are no strong variations in divergence as a function of the beam energy. (b) Ion current as a function of the beam energy.

the ion current as a function of the temperature of the Mo surface. The temperature is measured with an electrically isolated thermocouple which is not shown in Figure 1. As expected for thermal desorption, the ion current is increased at higher temperature.

To determine the beam size, i.e., its transversal section, we changed the position of the detector (screen) relative to the incident beam transversally. Figure 3(a) shows the ion current as a function of the relative position of the detector, for 4 different values of the beam energy $(25,50,75$, and $100 \mathrm{eV})$. The dashed lines in Figure 3(a) are fits of a Sigmoid function. From the values in Figure 3(a), one can estimate the effective width of the beam at the position of the detector to be $5 \mathrm{~mm}$, or about $2 \mathrm{msr}$. This implies that there are no strong variations of divergence as a function of the applied bias potential.

Figure 3(b) shows the dependence of ion current as a function of the acceleration potential. For potentials below $50 \mathrm{~V}$, oscillations in the current (two maxima) were observed. Probably, for these low potentials, there is a lensing effect, altering the position of the ion beam focus and consequently the ion current. For the region between 100 and $150 \mathrm{eV}$, almost linear behavior was obtained for the ion current. 
(a)

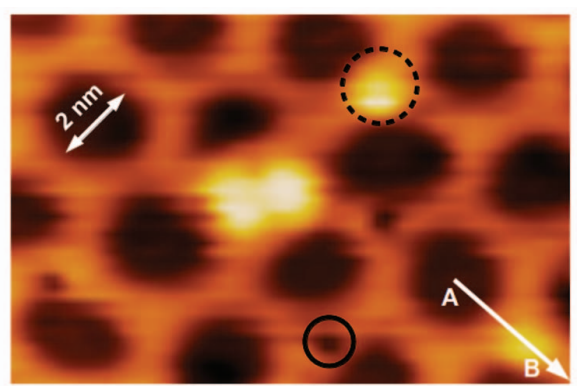

(b)

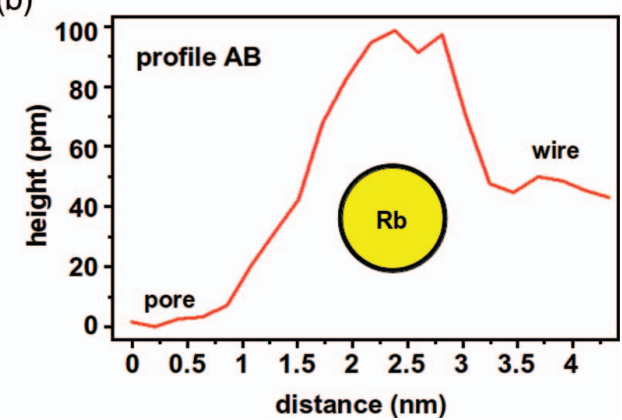

FIG. 4. (a) Scanning Tunneling Microscopy image $(13.5 \mathrm{~nm} \times 9 \mathrm{~nm})$ of $\mathrm{Rb}$ atoms implanted beneath the h-BN nanomesh, $V_{t}=2.0 \mathrm{~V}, I_{t}=0.1, \mathrm{nA}$ and $\mathrm{T}=34 \mathrm{~K}$. Rb atoms prefer to stay at the points of intersection of the wires (dashed circle). It is also possible to observe the vacancy defects generated by the penetration of the h-BN by the ions (solid circle). (b) Height Profile along the arrow $\mathrm{AB}$ in image (a).

Figure 4 shows the first application of the ion source for implantation of $\mathrm{Rb}$ atoms beneath the h-BN/Rh(111) nanomesh. ${ }^{4}$ The nanomesh is a superstructure formed on 12 $\times 12$ unit cells of $\mathrm{Rh}$ coinciding with $13 \times 13$ unit cells of h-BN. ${ }^{15,16}$ This corrugated monolayer of h-BN shows a superlattice parameter of $3.2 \mathrm{~nm}$. It consists of strongly coupled $\mathrm{h}$-BN regions to the $\mathrm{Rh}$ substrate (pores) and weakly coupled regions (wires). The pores have diameters of approximately $2 \mathrm{~nm}$ (see Figure 4) which may, for example, be used to accommodate molecules. ${ }^{2,17}$ The recipe for the preparation of $\mathrm{h}-\mathrm{BN} / \mathrm{Rh}$ nanomesh may be obtained from Ref. 18.

The implantation of $\mathrm{Rb}^{+}$ions with $100 \mathrm{eV}$ kinetic energy beneath the h-BN nanomesh is shown in Figure 4(a). We can see $4 \mathrm{Rb}$ related protrusions located at the intersection of the wires (dashed circle), which is similar to implanted Ar atoms. ${ }^{8}$ Two such nanotents are stabilized near the same site. Vacancy defects generated by the penetration of the ions through the h-BN (solid circle) are also observed. Figure 4(b) shows a height profile along the arrow AB in Figure 4(a). At a tunneling voltage of $2 \mathrm{~V}$, the height of protrusion is $\sim 1 \AA$.

This is smaller than that for the case of argon (1.6 ̊), which is taken as an indication that $\mathrm{Rb}$ maintains ionic character in the nanotent.

To summarize, we present the construction and performance of an alkali metal ion source. This source allows the acceleration of ions to an energy that makes it possible for them to penetrate one monolayers of graphene or hexagonal boron nitride. The ion current was demonstrated to be precisely controlled by the temperature of the ionizer surface. Scanning tunneling microscopy measurements demonstrate the efficiency of the source.

We thank Jean-Nicolas Longchamp for experimental help with the Pt sputter coating and the Swiss National Science Foundation for financial support. L. H. de Lima acknowledges a fellowship from the National Council for Scientific and Technological Development - CNPq (Brazil) and the hospitality of the University of Zürich.

${ }^{1}$ A. T. N'Diaye, S. Bleikamp, P. J. Feibelman, and T. Michely, Phys. Rev. Lett. 97, 215501 (2006).

${ }^{2}$ H. Dil, J. Lobo-Checa, R. Laskowski, P. Blaha, S. Berner, J. Osterwalder, and T. Greber, Science 319, 1824 (2008).

${ }^{3}$ L. H. de Lima, A. de Siervo, R. Landers, G. A. Viana, A. M. B. Goncalves, R. G. Lacerda, and P. Häberle, Phys. Rev. B 87, 081403(R) (2013).

${ }^{4}$ M. Corso, W. Auwärter, M. Muntwiler, A. Tamai, T. Greber, and J. Osterwalder, Science 303, 217 (2004).

${ }^{5}$ H. G. Zhang, J. T. Sun, T. Low, L. Z. Zhang, Y. Pan, Q. Liu, J. H. Mao, H. T. Zhou, H. M. Guo, S. X. Du, F. Guinea, and H.-J. Gao, Phys. Rev. B 84, 245436 (2011).

${ }^{6}$ T. Brugger, H. Ma, M. Iannuzzi, S. Berner, A. Winkler, J. Hutter, J. Osterwalder, and T. Greber, Angew. Chem., Int. Ed. 49, 6120 (2010).

${ }^{7}$ C. Riedl, C. Coletti, T. Iwasaki, A. A. Zakharov, and U. Starke, Phys. Rev. Lett. 103, 246804 (2009)

${ }^{8}$ H. Cun, M. Iannuzzi, A. Hemmi, S. Roth, J. Osterwalder, and T. Greber, Nano Lett. 13, 2098 (2013).

${ }^{9}$ S. Standop, O. Lehtinen, C. Herbig, G. Lewes-Malandrakis, F. Craes, J. Kotakoski, T. Michely, A. V. Krasheninnikov, and C. Busse, Nano Lett. 13, 1948 (2013).

${ }^{10}$ F. Banhart, Rep. Prog. Phys. 62, 1181 (1999).

${ }^{11}$ J. Kotakoski, C. H. Jin, O. Lehtinen, K. Suenaga, and A. V. Krasheninnikov, Phys. Rev. B 82, 113404 (2010).

${ }^{12}$ T. Greber, K. Freihube, R. Grobecker, A. Bottcher, K. Hermann, G. Ertl, and D. Fick, Phys. Rev. B 50, 8755 (1994).

${ }^{13}$ G. D. Alton, M. T. Johnson, and G. D. Mills, Nucl. Instrum. Methods Phys. Res. A 328, 154 (1993).

${ }^{14} \mathrm{See}$ http://www.saesgetters.com/products/alkali-metals-dispensers for more detailed information about Alkali Metal Dispensers.

${ }^{15}$ R. Laskowski, P. Blaha, T. Gallauner, and K. Schwarz, Phys. Rev. Lett. 98, 106802 (2007).

${ }^{16} \mathrm{~S}$. Berner, M. Corso, R. Widmer, O. Groening, R. Laskowski, P. Blaha, K. Schwarz, A. Goriachko, H. Over, S. Gsell, M. Schreck, H. Sachdev, T. Greber, and J. Osterwalder, Angew. Chem., Int. Ed. 46, 5115 (2007).

${ }^{17}$ H. Ma, T. Brugger, S. Berner, Y. Ding, M. Iannuzzi, J. Hutter, J. Osterwalder, and T. Greber, ChemPhysChem 11, 399 (2010).

${ }^{18}$ A. Goriachko, Y. He, M. Knapp, H. Over, M. Corso, T. Brugger, S. Berner, J. Osterwalder, and T. Greber, Langmuir 23, 2928 (2007). 